\title{
Covering Invariants and Cohopficity of 3-manifold Groups
}

\author{
Shicheng Wang ${ }^{1}$ and Ying-Qing $\mathrm{Wu}^{2}$
}

\begin{abstract}
A 3-manifold $M$ is called to have property $\mathrm{C}$ if the degrees of finite coverings over $M$ are determined by the manifolds. Thurston asked the problems of whether there is a real valued invariant of 3 -manifolds with a certain covering property, and what 3-manifolds have property $\mathrm{C}$. For geometric manifolds the conjecture is that $M$ has property $C$ if and only if it is not covered by surface $\times S^{1}$ or torus bundle over $S^{1}$. Using the Gromov norm, the conjecture is quickly reduced to the case of graph manifolds.

In this paper we define a new invariant $\omega(M)$ for graph manifolds. It has the covering property that if $N$ is a $d$-fold covering space of $M$, then $\omega(N)=d \omega(M)$. Thus in the generic case, when $\omega(M) \neq 0$, the manifold $M$ has property $\mathrm{C}$. We also use the methods to prove two other results. The first classifies all geometric manifolds that admit nontrivial self coverings. The second solves a problem of Gonzales-Acuna and Whitten about the cohopficity of 3-manifold groups, and determines all closed geometric manifolds that have cohopfian fundamental groups.
\end{abstract}

Definition. A 3-manifold $M$ is called to have property $\mathrm{C}$ if, whenever $M_{1}, M_{2}$ are homeomorphic covering spaces of $M$, the degrees of the coverings are the same. It has property $C_{r}$ if the above is true for all regular coverings.

We are interested in the following problems of Thurston:

(1) $[7$, Problem 3.16] Is there a reasonable real valued invariant $C(M)$ such that (a) if $M=M_{1} \# M_{2}$ then $C(M)=C\left(M_{1}\right)+C\left(M_{2}\right)$; (b) the covering property holds, i.e, if $M_{1}$ is a $k$-fold covering of $M$, then $C\left(M_{1}\right)=k C(M)$ ? $C(M)$ may have some additional properties.

(2) $[7$, Problem 3.16(a)] What 3-manifolds have property C?

An invariant of 3-manifolds with the covering property will give a partial solution to the Property $\mathrm{C}$ problem: if $C(M) \neq 0$, then the covering degree of $M_{1} \rightarrow M$ is given by $k=C\left(M_{1}\right) / C(M)$, so $M$ has property $\mathrm{C}$. There are some well known covering invariants. The simplest one is the number of 2-spheres on the boundary of an orientable manifold, and the most interesting one is the Gromov norm $\nu(M)$ defined by Gromov [3] soon after these questions were posed. The following definition is convenient for our purpose.

\footnotetext{
${ }^{0}$ Mathematics Subject Classification: 57M10, 57N10

${ }^{1}$ Partially supported by NSF of China

${ }^{2}$ Partially supported by NSF grant DMS 9102633
} 
Definition. A compact, connected, orientable 3-manifold $M$ is called geometric, if $M$ is either a Seifert manifold, or a hyperbolic manifold, or a Haken manifold, or a connected sum of such manifolds.

For a closed geometric 3-manifold $M, \nu(M)$ is essentially the sum of the volumes of all hyperbolic pieces in the geometric decomposition of $M$; therefore $M$ has property $\mathrm{C}$ if it contains some hyperbolic pieces. Note that the Gromov norm is a zero function on the set of all closed 3-manifolds which contain no hyperbolic pieces.

We only need to consider orientable manifolds, for if $M$ is a nonorientable 3-manifold, then it has property $\mathrm{C}$ if and only if its orientable double covering does. So we make the following

Convention. Except in section 8, all 3-manifolds in this paper are assumed orientable.

A famous conjecture is that all compact 3-manifolds are geometric. Also, it is known that if $M$ is covered by (surface) $\times S^{1}$ or torus bundle over $S^{1}$, then it does not have property C. Actually such manifolds admit nontrivial self coverings (see Theorem 8.6). Therefore we will concentrate on 3-manifolds in the class $\mathcal{G}$ defined below.

Notation. We use $\mathcal{G}$ to denote the set of all geometric 3-manifolds which are not covered by either (surface) $\times S^{1}$ or torus bundle over $S^{1}$.

\section{Conjecture 1: Every 3-manifold $M$ in $\mathcal{G}$ has Property $C$.}

A geometric 3-manifold $M$ is called a graph manifold if it has a nonempty canonical splitting surface (see section 1) cutting $M$ into Seifert manifold pieces. It can be shown (Thm 2.5) that Conjecture 1 is true if $M$ is not a graph manifold, so the problem is reduced to whether graph manifolds have property $\mathrm{C}$.

The main result of this paper is to define a new invariant $\omega(M)$ for graph manifolds, which takes values in $\mathbf{R}$. It is defined in terms of the Euler characteristics of orbifolds of the Seifert fibered pieces of $M$, and the intersection numbers of fibers on splitting surfaces. The central feature of $\omega(M)$ is the covering property. Hence in the generic case, when $\omega(M)$ is nonzero, $M$ has property $\mathrm{C}$.

A closely related problem is the cohopficity of 3-manifold groups. A group is called cohopfian if it is not isomorphic to a proper subgroup of itself. In [1] Gonzales-Acuna and Whitten studied this problem, and determined the cohopficity of the fundamental groups 
of Haken manifolds whose boundary is a nonempty union of tori. In section 8 we will show that the fundamental group of an irreducible closed geometric 3-manifold is cohopfian if and only if it is not covered by (surface) $\times S^{1}$ or torus bundle over $S^{1}$.

The paper is organized as follows. In section 1 we will review some basic definitions and properties about canonical splitting surfaces and Seifert fibered spaces. In section 2 we reduce Conjecture 1 to the problem of whether graph manifolds have property $\mathrm{C}$. Section 3 is devoted to the study decomposing surfaces, and section 4 to that of the fiber intersection numbers and their basic properties. In section 5 we use these informations to define weighted graphs for all nontrivial graph manifolds, and deduce a relation between the covering degree and the weights of the graph of the manifolds (Proposition 5.1). These are used in section 6 to define a matrix invariant $B(M)$, from which we will define the $\omega$-invariant, and prove its covering property (Theorem 6.6). We give some examples in section 7. The matrix $B(M)$ contains much more information. For example, it will be used to show that a nontrivial graph manifold has no nontrivial covering over itself (Proposition 8.2). We will also show in section 8 that a geometric manifold admits a nontrivial self covering if and only if it is covered by (surface) $\times S^{1}$ or torus bundle over $S^{1}$ (Theorem 8.6). This will be used to solve the cohopficity problem of Gonzales-Acuna and Whitten for closed geometric manifolds (Theorem 8.7). In section 9 we study the symmetry of the matrix $B(M)$, and show that all graph manifolds with at most 3 vertices have property $C_{r}$.

\section{$1 \quad$ Preliminaries}

Readers are referred to $[5,4]$ for background material on 3-manifold theory, to $[5,4,9]$ for definitions and basic results on Seifert fibered spaces, and to [9] for definitions and properties about orbifolds of Seifert fibered spaces and their Euler characteristics.

For a surface $S$ in a 3-manifold $M$, we use $N(S)$ to denote a regular neighborhood of $S$, and use $\eta(S)$ to denote the interior of $N(S)$. A 3-manifold with boundary a (possibly empty) union of tori is called simple if every embedded incompressible torus in $M$ is isotopic to a boundary component. The following are some results needed below.

Given a Haken manifold $M$ with $\partial M$ empty or a union of tori, there exists a surface $\mathcal{T}$, unique up to isotopy, satisfying the following conditions:

(a) Each component of $\mathcal{T}$ is a torus; 
(b) Each component of $M-\eta(\mathcal{T})$ is either a simple manifold or a Seifert fibered space;

(c) $\mathcal{T}$ is minimal subject to (a) and (b).

This surface $\mathcal{T}$ is called a canonical splitting surface [6].

Given a Seifert fibration on $M$, let $O M$ be the orbifold of $M$. Each regular fiber corresponds to a regular point in $O M$, and each singular fiber of type $(p, q)$ corresponds to a singular point of index $p$. The Euler characteristic of $O M$ is denoted by $\chi(O M)$. It is a rational number.

We say that a Seifert fibered space $M$ has hyperbolic orbifold if $\chi(O M)<0$. In this case, the Seifert fibration on $M$ is unique up to isotopy [9, Theorem 3.9]. If $\varphi: \tilde{M} \rightarrow M$ is a covering map, and $\chi(O M)<0$, then the number of fibers in $\tilde{M}$ that cover a regular fiber in $M$ is equal to $\chi(O \tilde{M}) / \chi(O M)$.

When $M$ has nonempty boundary and is not a solid torus, $\chi(O M) \leq 0$. If $\chi(O M)=0$, $M$ is either a product $I$-bundle over a torus or a twisted $I$-bundle over a Klein bottle.

Lemma 1.1 If $M$ is a twisted I-bundle over a Klein bottle, it has exactly two Seifert fibrations up to isotopy.

Proof. It is well known that there are two different Seifert fibrations on $M$, see for example [5, p87]. So we need only to show that there are at most two fibrations up to isotopy.

Consider a double covering $\psi: N \rightarrow M$, where $N$ is a (torus) $\times S^{1}$. Let $f: N \rightarrow N$ be the nontrivial covering transformation. Choose generators $c_{1}, c_{2} \in H_{1}(N)$ so that $f\left(c_{1}\right)=c_{1}$, and $f\left(c_{2}\right)=-c_{2}$. Suppose $\xi$ is a fibration of $M$, and let $\tilde{\xi}$ be its lifting to $N$. Consider a fiber $\alpha$ of $\xi$ on $\partial M$. It lifts to two fibers $\tilde{\alpha}_{1}, \tilde{\alpha}_{2}$ of $\tilde{\xi}$ on $\partial N$. As nonsingular fibers, $\tilde{\alpha}_{1}$ and $\tilde{\alpha}_{2}$ must be isotopic. Suppose $\tilde{\alpha}_{1}$ represents $a c_{1}+b c_{2}$ in $H_{1}(N)$. Then $\tilde{\alpha}_{2}=f\left(\tilde{\alpha}_{1}\right)=a c_{1}-b c_{2}$. As $\tilde{\alpha}_{1}$ and $\tilde{\alpha}_{2}$ are isotopic, $a=0$ or $b=0$. In other words, $\tilde{\alpha}_{1}=c_{1}$ or $c_{2}$. Therefore, $\alpha=\psi\left(c_{1}\right)$ or $\psi\left(c_{2}\right)$. The result now follows because Seifert fibrations are determined by fibers on the boundary, see [5, Lemma VI.19].

\section{Reduction to graph manifolds}

Lemma 2.1 Conjecture 1 is true if every manifold in $\mathcal{G}$ has property $C_{r}$.

Proof. Consider two covering maps $p_{1}, p_{2}: N \rightarrow M$. Pick up a subgroup $G \subset p_{1 *} \pi_{1}(N) \cap$ $p_{2 *} \pi_{1}(N)$ which is normal and has finite index in $\pi_{1}(M)$, and let $\tilde{M}$ be the covering space 
corresponding to $G$. Then the inclusion maps of $G$ into $p_{1 *} \pi_{1}(N), p_{2 *} \pi_{1}(N)$ and $\pi_{1}(M)$ induce finite regular coverings $q_{1}, q_{2}: \tilde{M} \rightarrow N$ and $p: \tilde{M} \rightarrow M$ such that $p=p_{1} q_{1}=p_{2} q_{2}$. By assumption $N$ has property $C_{r}$, so the degrees of $q_{1}$ and $q_{2}$ are the same. It follows that $p_{1}$ and $p_{2}$ also have the same degree.

By Lemma 2.1, in the text below we need only consider regular coverings.

Lemma 2.2 A 3-manifold $M$ in $\mathcal{G}$ admits a finite normal covering $\tilde{M}$ which has torsion free fundamental group.

Proof. Let $P_{1}, \ldots, P_{k}$ be the prime factors of $M$ which have finite fundamental groups. Recall that if a prime factor of $M$ has infinite fundamental group, then it is torsion free [4, Cor 9.9]. Thus any element of finite order in $\pi_{1}(M)$ is conjugate to an element in $\pi_{1}\left(P_{i}\right)$ for some $i$. In particular, $\pi_{1}(M)$ has only finitely many elements of finite order up to conjugacy.

Since $\pi_{1}(M)$ is residually finite (see $[10,3.3]$ ), for each torsion element $x$ there is a normal subgroup $G_{x}$ of finite index which does not contain $x$ (and hence any conjugate of $x)$. The intersection of all these $G_{x}$ is a finite intersection, and hence contains a normal subgroup $G$ which is of finite index in $\pi_{1}(M)$, and is torsion free. Let $\tilde{M}$ be the finite covering of $M$ corresponding to the subgroup $G \subset \pi_{1}(M)$. Then $\tilde{M}$ is as required.

Lemma 2.3 Suppose a non-prime geometric 3-manifold $M$ has torsion free fundamental group, and suppose $\partial M$ contains no 2-spheres. Then $M$ has property $C$.

Proof. Let $p: \tilde{M} \rightarrow M$ be a finite covering with degree $d$.

Since $\pi_{1}(M)$ contains no elements of finite order and $\partial M$ contains no 2-sphere components, we may assume that the prime factorization of $M$ consists of $c_{1}$ aspherical prime factors and $c_{2} S^{2} \times S^{1}$ prime factors; therefore there are $c(M)=c_{1}+c_{2}-1$ factorization 2 -spheres. Denoted by $S$ the union of these spheres. Suppose the prime factorization of $\tilde{M}$ consists of $c(\tilde{M})$ factorization 2-spheres.

Now $p^{-1}(S)$ is a union of $d c(M)$ two spheres. Since the prime factorization of $M$ contains no prime factors with finite fundamental group, no component of the closure of $\tilde{M}-p^{-1}(S)$ is a punctured 3-sphere. Hence $p^{-1}(S)$ is a family of factorization 2-spheres of $\tilde{M}$. By the uniqueness of prime factorization, we have $d c(M)=c(\tilde{M})$. Since $M$ is 
non-prime, $c(M)$ is not zero. Therefore,

$$
d=\frac{c(\tilde{M})}{c(M)}
$$

which is uniquely determined by $\tilde{M}$ and $M$.

Remark. The fact that $P^{3} \# P^{3}$ is an $n$-fold covering over itself for all $n>0$ shows that the formula above does not apply to the case when $M$ contains prime factors with finite fundamental groups. Here $P^{3}$ is the real projective 3 -space.

Theorem 2.4 Any non-prime 3-manifold $M$ in $\mathcal{G}$ has property $C$.

Proof. If $\partial M$ contains 2-sphere components, then the covering degree of $N \rightarrow M$ is the number of 2 -spheres in $\partial N$ divided by the number of 2 -spheres in $\partial M$. So we may assume $\partial M$ has no sphere components. Suppose there are coverings $p_{1}, p_{2}: N \rightarrow M$ such that the degrees of $p_{1}$ and $p_{2}$ are not the same. By the argument in proving Lemma 2.1, there are finite regular coverings $q_{1}, q_{2}: \tilde{N} \rightarrow N$ such that the degrees of $q_{1}$ and $q_{2}$ are not the same. By Lemma 2.2, after passing to a common regular covering of $M$ and $\tilde{N}$ if necessary, we may assume that $\pi_{1}(\tilde{N})$ has no torsion elements. For the same reason as above, there are coverings $r_{1}, r_{2}: \tilde{M} \rightarrow \tilde{N}$ such that the degrees of $r_{1}$ and $r_{2}$ are not equal. By Lemma 2.3, this could happen only if $\tilde{N}$ is a prime 3-manifold; so either it is an $S^{1} \times S^{2}$, or it is irreducible. But the first case implies that $M$ is not in $\mathcal{G}$ by definition, and the second case contradicts the fact that $\pi_{2}(\tilde{N})=\pi_{2}(M) \neq 0$.

Theorem 2.5 If a 3-manifold $M$ in $\mathcal{G}$ is not a graph manifold, then $M$ has property $C$.

Proof. Let $\tilde{M} \rightarrow M$ be a regular covering map of degree $d$.

By Theorem 2.4, we may assume $M$ is prime. In particular $\partial M$ contains no 2-sphere components. If $\chi(\partial M)<0$, then $\chi(\partial \tilde{M})<0$ and $d=\chi(\partial \tilde{M}) / \chi(\partial M)$. So we may assume that $\chi(\partial M)=0$. Thus $\partial M$ consists of a union of tori (possibly an empty set). The canonical splitting surface (see $\S 1$ ) cuts $M$ into simple manifolds and Seifert fibered spaces. By Thurston's theorem, a simple manifold admits a hyperbolic structure, which is unique up to isometries. Let $v(M)$ be the sum of the volumes of the hyperbolic pieces. Then it is an invariant of $M$, and it has the covering property. Therefore, if $v(M) \neq 0$, then $M$ has property $\mathrm{C}$. 
Now we consider the case when $M$ is a Seifert manifold.

If the Euler number $e(M)$ of $M$ is zero (see [9, Section 3] for definitions), or if the Euler characteristic $\chi(O M)$ of the orbifold of $M$ is zero, then it is known that $M$ is finitely covered by either (surface) $\times S^{1}$ or a torus bundle over $S^{1}$. In this case, $M$ is not in $\mathcal{G}$.

If $e(M) \neq 0$ and $\chi(O M)>0$, then $M$ is covered by $S^{3}$. So $\pi_{1}(M)$ is finite, and for any covering $p$ from $\tilde{M}$ to $M$, the degree of $p$ is equal to $\left|\pi_{1}(M)\right| /\left|\pi_{1}(\tilde{M})\right|$.

If $e(M) \neq 0$ and $\chi(O M)<0$, then $M$ admits a unique Seifert fibration. So we may assume that $p$ is fiber preserving. Let the orbifold covering degree and the fiber covering degree of $p$ be $l$ and $m$ respectively. Then $d=m l$, and $l=\chi(O \tilde{M}) / \chi(O M)$. By Theorem 3.6 of [9], we have

$$
e(\tilde{M})=\frac{e(M) l}{m}
$$

So we have

$$
d=\frac{l^{2} e(M)}{e(\tilde{M})}=\frac{\chi(O \tilde{M})^{2} e(M)}{\chi(O M)^{2} e(\tilde{M})}
$$

Therefore $M$ has property C. Clearly, $C(M)=\chi(O M)^{2} / e(M)$ is an invariant with the covering property.

The remaining case is when $M$ has a nonempty canonical splitting surface, and all pieces are Seifert fibered spaces. By definition $M$ is a graph manifold.

\section{Decomposing surfaces of graph manifolds}

The canonical splitting surface of $M$ cuts $M$ into Seifert fibered pieces. But from our point of view, it has two disadvantages:

(1) It is not preserved by covering maps. If $\varphi: \tilde{M} \rightarrow M$ is a covering map, and $\mathcal{T}$ is the canonical splitting surface in $M$, then $\varphi^{-1}(\mathcal{T})$ is usually not a canonical splitting surface for $\tilde{M}$.

(2) Seifert fibrations on $M-\eta(\mathcal{T})$ may not be unique.

These problems happen when some component $T$ of $\mathcal{T}$ bounds a twisted $I$-bundle over a Klein bottle. It is possible that $T$ bounds twisted $I$-bundles on both sides, or that the complement of $\eta(T)$ is a (torus) $\times I$. We call such a manifold a trivial graph manifold. Note that a trivial graph manifold is closed, and is covered by a torus bundle over $S^{1}$. Since it is not Seifert fibered, by [9, Theorem 5.3], it admits a Sol geometry. On the other hand, if $M$ is a nontrivial graph manifold, then some Seifert fibered piece of $M$ has a hyperbolic 
orbifold, so it is not covered by torus bundle over $S^{1}$. Therefore, a graph manifold is trivial if and only if it is covered by some torus bundle over $S^{1}$.

Now consider a nontrivial graph manifold $M$. We define a decomposing surface of $M$ to be a surface $\mathcal{S}$, which is the union of those tori in $\mathcal{T}$ that do not bound twisted $I$-bundles, and the central Klein bottles in the twisted $I$-bundle components of $M-\eta(T)$. In other words, we replace each torus $T$ which bounds a twisted $I$-bundle $N$ by the central Klein bottle in $N$. The following lemma follows from the above definition and the uniqueness of canonical splitting surfaces.

Lemma 3.1 If $M$ is not covered by a torus bundle over $S^{1}$, then it has a decomposing surface, which is unique up to ambient isotopy.

Consider a canonical splitting surface $\mathcal{T}$ of $M$. Let $\mathcal{S}$ be the corresponding decomposing surface. Notice that a twisted $I$-bundle over Klein bottle is a regular neighborhood of its central Klein bottle, so it is easy to see that $M-\eta(\mathcal{S})$ can be obtained from $M-\eta(T)$ by deleting the twisted $I$-bundle components. Therefore each component of $M-\eta(\mathcal{S})$ has hyperbolic orbifold.

On the other hand, if we have a surface $\mathcal{S}$ in $M$ consisting of tori and Klein bottles, such that $M-\eta(\mathcal{S})$ consists of Seifert fibered spaces with hyperbolic orbifolds, then we can construct a union of tori $\mathcal{T}$ by reversing the above process, i.e $\mathcal{T}$ is the union of the tori in $\mathcal{S}$ and the boundary of the regular neighborhood of Klein bottles in $\mathcal{S}$. It is clear that $M-\eta(\mathcal{T})$ is a Seifert fibered space. Moreover, $M-\eta\left(\mathcal{T}^{\prime}\right)$ is Seifert fibered for some proper subset $\mathcal{T}^{\prime}$ of $\mathcal{T}$ if and only if $M-\eta\left(\mathcal{S}^{\prime}\right)$ is Seifert fibered for some proper subset $\mathcal{S}^{\prime}$ of $\mathcal{S}$. Therefore, from the definition of canonical splitting surfaces we have the following characterization of decomposing surfaces.

Lemma 3.2 A surface $\mathcal{S}$ in $M$ is a decomposing surface if and only if

(a) $\mathcal{S}$ is a union of tori and Klein bottles;

(b) $M-\eta(\mathcal{S})$ consists of Seifert fibered spaces with hyperbolic orbifolds;

(c) $\mathcal{S}$ is minimal subject to (a) and (b).

\section{Fiber intersection number}

Consider $N=T \times I$, a trivial $I$-bundle over a torus $T$. Suppose $\partial N$ has been given an $S^{1}$

fibration structure $\xi$. Choose a fiber $\alpha_{i}$ on $T \times i$ for $i=0,1$, and let $\alpha_{1}^{\prime}$ be the curve on 
$T \times 0$ which is isotopic to $\alpha_{1}$ in $N$. We define the fiber intersection number on $T$ as

$$
\Delta(T)=\Delta_{\xi}(T)=\left|\alpha_{0} \cdot \alpha_{1}^{\prime}\right|
$$

where $\alpha_{0} \cdot \alpha_{1}^{\prime}$ is the algebraic intersection number of the two curves on $T \times 0$.

Suppose $\varphi: \tilde{N} \rightarrow N$ is a $k$-fold covering map. Then $\tilde{N}$ is also a trivial $I$-bundle over a torus $\tilde{T}$. Endow $\partial \tilde{N}$ with the $S^{1}$ fibration structure induced by $\varphi$.

Lemma 4.1 Let $s_{i}$ be the number of fibers in $\partial \tilde{N}$ that cover a fiber in $T \times i$. Then

$$
s_{0} s_{1} \Delta(\tilde{T})=k \Delta(T)
$$

Proof. Choose a fiber $\alpha_{i}$ on $T \times i$. It lifts to a union $\tilde{\alpha}_{i}$ of fibers. Let $\beta_{i}$ be a component of $\tilde{\alpha}_{i}$. Clearly, $\tilde{\alpha}_{1}$ is isotopic to the lifting $\tilde{\alpha}_{1}^{\prime}$ of $\alpha_{1}^{\prime}$. Each intersection point of $\alpha_{0}$ and $\alpha_{1}^{\prime}$ lifts to $k$ points of intersection of $\tilde{\alpha}_{0}$ and $\tilde{\alpha}_{1}^{\prime}$, so we have

$$
\tilde{\alpha}_{0} \cdot \tilde{\alpha}_{1}^{\prime}=k\left(\alpha_{0} \cdot \alpha_{1}^{\prime}\right)
$$

Since $\tilde{\alpha}_{i}$ consists of $s_{i}$ copies of $\beta_{i}$, we have

$$
\tilde{\alpha}_{0} \cdot \tilde{\alpha}_{1}^{\prime}=s_{0} s_{1}\left(\beta_{0} \cdot \beta_{1}^{\prime}\right)
$$

By definition, $\Delta(\tilde{T})=\left|\beta_{0} \cdot \beta_{1}^{\prime}\right|$. Therefore, $s_{0} s_{1} \Delta(\tilde{T})=\left|\tilde{\alpha}_{0} \cdot \tilde{\alpha}_{1}^{\prime}\right|=k \Delta(T)$.

Now suppose $N$ is a twisted $I$-bundle over a Klein bottle $K$. Then $\partial N$ is a single torus. Up to isotopy $N$ has exactly two Seifert fibered structures (Lemma 1.1). We denote by $c_{1}$ a curve on $\partial N$ that is a fiber of the fibration whose orbifold is a Möbius band, and denote by $c_{2}$ a fiber on $\partial N$ of the other fibration. They form a coordinate system on $\partial N$. Given an arbitrary $S^{1}$ fibration $\xi$ on $\partial N$, a fiber $\alpha$ of $\xi$ represents a unique element $a c_{1}+b c_{2}$ in $H_{1}(\partial N)$. We define the fiber intersection number on the Klein bottle $K$ to be

$$
\Delta(K)=|4 a b|
$$

Consider a finite covering map $\varphi: \tilde{N} \rightarrow N . \tilde{N}$ is an $I$-bundle over a surface $\tilde{S}$, which is either a torus or a Klein bottle. Endow $\partial \tilde{N}$ with the induced $S^{1}$ fibration $\varphi^{-1}(\xi)$. We have

Lemma 4.2 Suppose $\varphi: \tilde{N} \rightarrow N$ is a regular covering. Let $s$ be the number of fibers in $\partial \tilde{N}$ that cover a fiber $\alpha$ of the $S^{1}$ fibration $\xi$ in $\partial N$. Then

$$
s^{2} \Delta(\tilde{S})=k \Delta(K)
$$


Proof. We divide the lemma into several cases.

CASE 1: $\tilde{S}$ is a Klein bottle.

In this case $\tilde{N}$ is a twisted $I$ bundle over $\tilde{S}$. Consider the lifting $\tilde{c}_{i}$ of $c_{i}$. Suppose $\tilde{c}_{i}$ has $t_{i}$ components, and let $\tilde{e}_{i}$ be one of them. Since the lifting of a Seifert fibration of $N$ is a Seifert fibration of $\tilde{N}$, we know that $\tilde{e}_{1}, \tilde{e}_{2}$ are fibers of the Seifert fibrations on $\tilde{N}$, so they form the required coordinate system. Since $\alpha$ represents $a c_{1}+b c_{2}$ in $H_{1}(\partial N)$, its lifting represents

$$
\tilde{\alpha}=a \tilde{c}_{1}+b \tilde{c}_{2}=\left(a t_{1}\right) \tilde{e}_{1}+\left(b t_{2}\right) \tilde{e}_{2} .
$$

Since $\tilde{\alpha}$ is a union of simple closed curves, the number of curves in $\tilde{\alpha}$ is $s=\operatorname{gcd}\left(a t_{1}, b t_{2}\right)$. Therefore each component $\beta$ of $\tilde{\alpha}$ represents

$$
\beta=\left(\frac{a t_{1}}{s}\right) \tilde{e}_{1}+\left(\frac{b t_{2}}{s}\right) \tilde{e}_{2}
$$

in $H_{1}(\partial \tilde{N})$. By definition,

$$
\Delta(\tilde{S})=\left|4\left(\frac{a t_{1}}{s}\right)\left(\frac{b t_{2}}{s}\right)\right|=\frac{t_{1} t_{2}}{s^{2}} \Delta(K) .
$$

The result now follows from the fact that the covering degree $k=t_{1} t_{2}$.

CASE 2: $\tilde{N}$ is the double covering corresponding to the map $\pi_{1}(\partial N) \rightarrow \pi_{1}(N)$ induced by the inclusion map.

In this case, $\tilde{S}$ is a torus, and $\tilde{N}=\tilde{S} \times I$. The restriction of $\varphi$ to each component of $\partial \tilde{N}$ is a homeomorphism. We use $c_{i}^{j}$ to denote the curve $\varphi^{-1}\left(c_{i}\right)$ on $\tilde{S} \times j, j=0,1$.

It is easy to see that $c_{1}^{0}$ is isotopic to $-c_{1}^{1}$ in $\tilde{N}$, while $c_{2}^{0}$ is isotopic to $c_{2}^{1}$. A fiber $\beta_{0}$ of $\varphi^{-1}(\xi)$ in $\tilde{S} \times 0$ represents $a c_{1}^{0}+b c_{2}^{0}$, while a fiber $\beta_{1}$ in $\tilde{S} \times 1$ represents $a c_{1}^{1}+b c_{2}^{1}$. Since in $\tilde{N}$ the curve $\beta_{1}$ is isotopic to $(-a) c_{1}^{0}+b c_{2}^{0}$, we have

$$
\Delta(\tilde{S})=\left|\operatorname{det}\left(\begin{array}{cc}
a & b \\
-a & b
\end{array}\right)\right|=|2 a b|=\frac{1}{2} \Delta(K) .
$$

Since in this case $k=s=2$, the result follows.

CASE 3: $\tilde{S}$ is a torus, $\varphi: \tilde{N} \rightarrow N$ is a map of degree $k>2$.

Any such map factors through a covering

$$
\tilde{N} \rightarrow \hat{N} \rightarrow N
$$

where $\hat{N}$ is the double covering in Case 2. Since $\tilde{N}$ is orientable, it is a product $\tilde{S} \times I$. By assumption, $\varphi$ is a regular covering, so there is a covering transformation sending $\tilde{S} \times 0$ 
to $\tilde{S} \times 1$. Thus the number of fibers in $\tilde{S} \times 0$ covering a fiber $\alpha$ on $\partial N$ is the same as that in $\tilde{S} \times 1$. Since the total number is $s$, each $\tilde{S} \times i$ has $s / 2$ fibers covering $\alpha$. Write $\hat{N}$ as $\hat{T} \times I$. Let $\beta_{i}$ be the curve in $\hat{T} \times i$ that covers $\alpha$. Then each $\beta_{i}$ is covered by $s / 2$ fibers in $\tilde{S} \times i$. By Lemma 3.1 and Case 2 above, we have

$$
\begin{aligned}
(s / 2)(s / 2) \Delta(\tilde{S}) & =(k / 2) \Delta(\hat{T}) \\
\Delta(\hat{T}) & =(1 / 2) \Delta(K)
\end{aligned}
$$

Combining these together yields $s^{2} \Delta(\tilde{S})=k \Delta(K)$, as required.

Lemma 4.3 Suppose $N$ is an I-bundle over $S$, where $S$ is either a torus or a Klein bottle. Let $\xi$ be an $S^{1}$ fibration on $\partial N$. Then $\xi$ extends to a Seifert fibration on $N$ if and only if $\Delta(S)=0$.

Proof. If $S$ is a torus, then the extension exists if and only if the fibers of $\xi$ on the two components of $\partial N$ are isotopic to each other, which is equivalent to $\Delta(S)=0$. If $S$ is a Klein bottle, then $N$ has exactly two fibrations up to isotopy, and $\xi$ extends to one of them if and only the $a$ or $b$ in the definition of $\Delta(S)$ is 0 .

Consider a graph manifold $M$. Let $\mathcal{S}$ be a surface satisfying (a) and (b) of Lemma 3.2. Then $M-\eta(\mathcal{S})$ has a unique Seifert fibration structure, which gives rise to an $S^{1}$ fibration structure on $\partial N(\mathcal{S})$, so each component $S$ of $\mathcal{S}$ has a well defined fiber intersection number $\Delta(S)$. The surface $\mathcal{S}$ satisfies (c) of Lemma 3.2 if and only if the fibration on $\partial N(\mathcal{S})$ cannot be extended to Seifert fibrations over any $N(S)$. By Lemma 4.3, this is true if and only $\Delta(S) \neq 0$ for all $S$ in $\mathcal{S}$.

Now suppose $\varphi: \tilde{M} \rightarrow M$ is a finite covering over a graph manifold $M$ which is not covered by torus bundles over $S^{1}$. Then $M$ has a nonempty decomposing surface $\mathcal{S}$. Let $\tilde{\mathcal{S}}$ be the surface $\varphi^{-1}(\mathcal{S})$ in $\tilde{M}$. Clearly it satisfies (a) of Lemma 3.2. Since $\tilde{M}-\eta(\tilde{\mathcal{S}})$ covers $M-\eta(\mathcal{S})$, it also satisfies (b) of Lemma 3.2. By Lemmas 4.1 and $4.2, \Delta(S) \neq 0$ for all $S$ in $\mathcal{S}$ if and only if $\Delta(\tilde{S}) \neq 0$ for all $\tilde{S}$ in $\tilde{\mathcal{S}}$. Therefore, $\tilde{\mathcal{S}}$ satisfies (c) of Lemma 3.2. So we have

Corollary 4.4 If $\mathcal{S}$ is a decomposing surface for $M$, then $\tilde{\mathcal{S}}=\varphi^{-1}(\mathcal{S})$ is a decomposing surface for $\tilde{M}$. 


\section{Graph of Manifolds}

Suppose $M$ is a nontrivial graph manifold. As we have seen above such a manifold has a decomposing surface $\mathcal{S}$. We define a graph $\Gamma(M)$ as follows: For each component $N_{i}$ of $M-\eta(\mathcal{S})$ is assigned a vertex $v_{i}$, and for each component $S_{j}$ of $\mathcal{S}$ is assigned an edge $e_{j}$, so that

(1) If $S_{j}$ is a torus, and $\partial N\left(S_{j}\right)$ has one component in each of $N_{i}$ and $N_{k}$ (i may be equal to $k$ ), then $e_{j}$ has endpoints on $v_{i}$ and $v_{k}$;

(2) If $S_{j}$ is a Klein bottle, and $\partial N\left(S_{j}\right)$ is in $N_{i}$, then $e_{j}$ has both endpoints on $v_{i}$.

We define a "weight" for each vertex or edge of $\Gamma(M)$ as follows. If $v_{i}$ is a vertex of $\Gamma(M)$ corresponding to a component $M_{i}$ of $M-\eta(\mathcal{S})$, let the weight $x_{i}=x\left(v_{i}\right)$ be the Euler characteristic of the orbifold of $N_{i}$. If $e$ is an edge corresponding to a surface $S$ in $\mathcal{S}$, let the weight $\Delta(e)$ be the fiber intersection number $\Delta(S)$. Since each component of $M-\eta(\mathcal{S})$ has hyperbolic orbifold, the weights of vertices are nonzero rational numbers. Also, by the remark after Lemma 4.3, the weights of edges are all nonzero integers. Since the decomposing surface and the Seifert fibrations on the pieces are unique, this weighted graph is well defined, and is an invariant of $M$.

Consider a covering map $\varphi: \tilde{M} \rightarrow M$. As we have seen before, if $\mathcal{S}$ is a decomposing surface for $M$, then $\tilde{\mathcal{S}}=\varphi^{-1}(\mathcal{S})$ is a decomposing surface for $\tilde{M}$. Therefore $\varphi$ induces a map on the graphs

$$
\varphi: \Gamma(\tilde{M}) \rightarrow \Gamma(M)
$$

It is defined in the obvious way. A vertex $\tilde{v}_{p}$ is mapped to $v_{i}$ if the corresponding component $\tilde{N}_{p}$ in $\tilde{M}-\eta(\tilde{\mathcal{S}})$ is mapped to $N_{i}$, and an edge $\tilde{e}_{q}$ is mapped to $e_{j}$ if the corresponding surface $\tilde{S}_{q}$ covers $S_{j}$.

We now assume $\varphi$ is a regular covering. The main result of this section is Proposition 5.1 below, which expresses the covering degree $d$ in terms of the weights of the graphs. We need the following notations.

For each vertex $v_{i}$ in $\Gamma(M)$, define

$$
I_{i}=\left\{p \mid \varphi\left(\tilde{v}_{p}\right)=v_{i}\right\}
$$

We use $u_{i}$ to denote the number of elements in $I_{i}$. In other words, $u_{i}$ is the number of components in $\tilde{M}-\eta(\tilde{\mathcal{S}})$ that covers the component $N_{i}$ in $M-\eta(\mathcal{S})$. 
Suppose $\tilde{v}_{p}, \tilde{v}_{q}$ are two vertices covering $v_{i}$ Since $\varphi$ is a regular covering, there is a covering transformation $f: \tilde{M} \rightarrow \tilde{M}$ which maps the component $\tilde{N}_{p}$ homeomorphically onto $\tilde{N}_{q}$. Therefore, $x\left(\tilde{v}_{p}\right)=x\left(\tilde{v}_{q}\right)$. Hence we can use $\tilde{x}_{i}$ to denote $x\left(\tilde{v}_{p}\right)$ without ambiguity.

Proposition 5.1 Let $e$ be an edge in $\Gamma(M)$ with endpoints on $v_{i}$ and $v_{j}$. Let $t$ be the number of edges in $\Gamma(\tilde{M})$ covering e, and let $\tilde{e}$ be one of these edges. Then

$$
d=\frac{\Delta(\tilde{e}) \tilde{x}_{i} \tilde{x}_{j} u_{i} u_{j}}{t \Delta(e) x_{i} x_{j}}
$$

Proof. Let $\tilde{v}_{p}, \tilde{v}_{q}$ be the endpoints of $\tilde{e}$. As before, we use $\tilde{N}_{p}, \tilde{N}_{q}$ to denote the corresponding components of $\tilde{M}-\eta(\tilde{\mathcal{S}})$. There are several cases.

CASE 1: The surface $S$ corresponding to $e$ is a torus.

Let $T_{i}, T_{j}$ be the tori on $\partial N(S)$, with $\alpha_{i}$ and $\alpha_{j}$ a fiber on $T_{i}$ and $T_{j}$ respectively. Denote by $\tilde{S}$ the torus in $\tilde{M}$ corresponding to $\tilde{e}$. By Lemma 4.1 we have

$$
s_{i} s_{j} \Delta(\tilde{S})=k \Delta(S)
$$

where $k$ is the covering degree of $\tilde{S} \rightarrow S$, and $s_{i}$ is the number of fibers on $\partial N(\tilde{S})$ that cover $\alpha_{i}$.

By definition $t$ is the number of edges in $\Gamma(\tilde{M})$ that is mapped to $e$, so equivalently it is the number of tori in $\tilde{M}$ covering $T_{i}$. Since there are $u_{i}$ vertices in $\Gamma(\tilde{M})$ covering $v_{i}$, there are $t / u_{i}$ tori in $\tilde{N}_{p}$ that cover $T_{i}$. The orbifold covering degree of $\tilde{N}_{p}$ over $N_{i}$ is $\tilde{x}_{i} / x_{i}$, so on each torus there are $\left(\tilde{x}_{i} / x_{i}\right) /\left(t / u_{i}\right)$ fibers covering $\alpha_{i}$. That is, $s_{i}=\tilde{x}_{i} u_{i} / t x_{i}$. Substitute this into equation (2), we have

$$
\tilde{x}_{i} \tilde{x}_{j} u_{i} u_{j} \Delta(\tilde{S})=k t^{2} x_{i} x_{j} \Delta(S)=d t x_{i} x_{j} \Delta(S) .
$$

\section{CASE 2: Both $S$ and $\tilde{S}$ are Klein bottles.}

The computation in this case is quite similar. We omit the details.

CASE 3: $S$ is a Klein bottle, $\tilde{S}$ is a torus.

Recall that $s_{i}=s_{j}$ is the number of fibers on $\partial N(\tilde{S})$ that cover a fiber $\alpha$ in $\partial N$. As the covering is regular, there is a covering transformation sending one torus of $\partial N(\tilde{S})$ to the other, so on each torus there are $s_{i} / 2$ fibers covering $\alpha$. Since each $\tilde{S}$ corresponds to 2 tori covering $\partial N$, there are $2 t$ tori in $\tilde{M}$ that cover $\partial$. Thus on each $\tilde{N}_{p}$ there are $2 t / u_{i}$ tori covering $\partial N$. Therefore, on each torus, there should be $\left(\tilde{x}_{i} / x_{i}\right) /\left(2 t / u_{i}\right)$ fibers covering 
$\alpha$. That is, $s_{i} / 2=\left(\tilde{x}_{i} / x_{i}\right) /\left(2 t / u_{i}\right)$. Hence $s_{i}=\tilde{x}_{i} u_{i} / x_{i} t$. Equation (3) now follows from Lemma 4.2 .

\section{The invariant}

Proposition 5.1 gives a formula about the degree of a covering map. However, it depends on $u_{i}, u_{j}$ and $t$, which usually depend on the covering. In this section we will simplify the formula, then obtain an invariant from a system of linear equations.

For each edge $e$ in the graph $\Gamma(M)$, define

$$
a(e)=\frac{1}{\Delta(e) x_{i} x_{j}}
$$

Then equation (1) can be rewritten as

$$
d t a(\tilde{e})=a(e) u_{i} u_{j}
$$

or

$$
d \sum a(\tilde{e})=a(e) u_{i} u_{j}
$$

where $\sum$ is taken over all $\tilde{e}$ that is mapped to $e$. For all $i, j$, define

$$
a_{i j}=a_{i j}(\Gamma(M))=\left\{\begin{array}{l}
\sum\left\{a(e) \mid e \text { has endpoints on } v_{i} \text { and } v_{j}\right\} \\
0 \quad \text { if there is no such } e
\end{array}\right.
$$

Then the equation (4), when sum over all such $e$, becomes

$$
d \sum a(\tilde{e})=a_{i j} u_{i} u_{j}
$$

where the sum is taken over all edges $\tilde{e}$ in $\Gamma(\tilde{M})$ that cover some $e$ connecting $v_{i}$ to $v_{j}$. Note that $\tilde{e}$ is such an edge if and only if it has endpoints on some $\tilde{v}_{p}$ and $\tilde{v}_{q}$, where $\tilde{v}_{p}$ is mapped to $v_{i}$, and $\tilde{v}_{q}$ is mapped to $v_{j}$. Use $\tilde{a}_{p q}$ to denote $a_{p q}(\Gamma(\tilde{M}))$. Then the above sum can be written as

$$
\begin{aligned}
\sum a(\tilde{e}) & =\sum\left\{\tilde{a}_{p q} \mid \tilde{v}_{p} \text { covers } v_{i}, \tilde{v}_{q} \text { covers } v_{j}\right\} \\
& =\sum\left\{\tilde{a}_{p q} \mid p \in I_{i}, q \in I_{j}\right\}
\end{aligned}
$$

When $i \neq j$, the two sets $I_{i}$ and $I_{j}$ are disjoint, so we have

$$
\sum\left\{\tilde{a}_{p q} \mid p \in I_{i}, q \in I_{j}\right\}=\sum_{p \in I_{i}} \sum_{q \in I_{j}} \tilde{a}_{p q} .
$$


When $i=j$, the two sets $I_{i}$ and $I_{j}$ are equal, so

$$
\sum\left\{\tilde{a}_{p q} \mid p \in I_{i}, q \in I_{j}\right\}=\frac{1}{2} \sum_{p \in I_{i}} \sum_{q \in I_{j}-\{p\}} \tilde{a}_{p q}+\sum_{p=q \in I_{i}} \tilde{a}_{p q}
$$

To simplify the formulas, we define

$$
b_{i j}= \begin{cases}a_{i j} & \text { if } i \neq j \\ 2 a_{i j} & \text { if } i=j\end{cases}
$$

Define $\tilde{b}_{p q}$ in the same way. Then the above equations (5), (6) (7) and (8) can be combined into

Theorem 6.1 Suppose $\varphi: \tilde{M} \rightarrow M$ is a regular covering over a nontrivial graph manifold. Then the degree of $\varphi$ satisfies the equation

$$
d \sum_{p \in I_{i}} \sum_{q \in I_{j}} \tilde{b}_{p q}=b_{i j} u_{i} u_{j}
$$

Definition Given a nontrivial graph manifold $M$, let $B=B(M)=\left(b_{i j}\right)$ be the matrix with $b_{i j}$ defined as above. Let $J=(1,1, \ldots, 1)^{T}$. The $\omega$-invariant of $M$ is defined by

$$
\omega(M)= \begin{cases}0 & \text { if } B y=J \text { has no solution } \\ \sum y_{i} & \text { if } B y=J \text { has a solution } y=\left(y_{1}, y_{2}, \ldots\right)^{T}\end{cases}
$$

Proposition $6.2 \omega(M)$ is an invariant of $M$.

Proof: Recall that the weighted graph $\Gamma(M)$ is an invariant of $M$. The definition of $B=B(M)$ depends only on $\Gamma(M)$ and the indexing of its vertices, so up to simultaneous permutations of rows and columns it is well defined. A row permutation of $B$ will not change the solutions to $B y=J$, while a column permutation of $B$ will result in a permutation of the components of the solutions, which has no affect on $\sum y_{i}$. Therefore the Proposition is true if either $B y=J$ has no solution or if the solution if unique. Now suppose $y, z$ are two solutions. Then

$$
B(y-z)=0, \text { and }\left(y^{T}-z^{T}\right) B=0
$$


Therefore

$$
\begin{aligned}
\sum y_{i} & =y^{T} J=y^{T} B y=\left(z^{T}+\left(y^{T}-z^{T}\right)\right) B(z+(y-z)) \\
& =z^{T} B z+z^{T} B(y-z)+\left(y^{T}-z^{T}\right) B y=z^{T} B z \\
& =\sum z_{i}
\end{aligned}
$$

Suppose $\tilde{M}$ is a covering space over $M$. As before, we use $\tilde{B}=B(\tilde{M})=\left(\tilde{b}_{p q}\right)$ to denote the corresponding matrix of $\tilde{M}$. Given $i, j$, and $q \in I_{j}$, define

$$
c_{i q}=\sum_{p \in I_{i}} \tilde{b}_{p q}
$$

We need the following

Lemma 6.3 If $\varphi: \tilde{M} \rightarrow M$ is a regular covering, then $c_{i q}$ is independent of the choice of $q \in I_{j}$.

Proof: Suppose $q, q^{\prime} \in I_{j}$. Since $\varphi$ is a regular covering, there is a covering transformation $f$ sending a component $\tilde{M}_{q}$ of $\tilde{M}-\eta(\tilde{S})$ to the component $\tilde{M}_{q^{\prime}}$. Suppose $f$ sends $\tilde{M}_{p}$ to $\tilde{M}_{p^{\prime}}$. Then an edge $e$ having endpoints on $\tilde{v}_{p}$ and $\tilde{v}_{q}$ is sent to the edge $f(e)$ with endpoints on $\tilde{v}_{p^{\prime}}$ and $\tilde{v}_{q^{\prime}}$. Since $f$ is a homeomorphism, it induces a weight preserving isomorphism of the graph $\Gamma(\tilde{M})$; so $a(e)=a(f(e))$. Summing over all edges connecting $\tilde{v}_{p}$ and $\tilde{v}_{q}$, we have $\tilde{a}_{p q}=\tilde{a}_{p^{\prime} q^{\prime}}$; therefore $\tilde{b}_{p q}=\tilde{b}_{p^{\prime} q^{\prime}}$. While $v_{p}$ ranges over all vertices with $p \in I_{i}$, $v_{p^{\prime}}=f\left(v_{p}\right)$ also ranges over all such vertices. Hence,

$$
\sum_{p \in I_{i}} \tilde{b}_{p q}=\sum_{p^{\prime} \in I_{i}} \tilde{b}_{p^{\prime} q^{\prime}}=\sum_{p \in I_{i}} \tilde{b}_{p q^{\prime}}
$$

Proposition 6.4 Suppose $\varphi: \tilde{M} \rightarrow M$ is a d-fold regular covering. If $\tilde{B} z=J$ has a solution, then so does $B y=J$, and $\omega(\tilde{M})=d \omega(M)$.

Proof: Since $c_{i q}$ is independent of $q \in I_{j}$, we may write it as $c_{i j}$. By Theorem 6.1, we have

$$
\begin{aligned}
b_{i j} u_{i} u_{j} & =d \sum_{p \in I_{i}} \sum_{q \in I_{j}} \tilde{b}_{p q}=d \sum_{q \in I_{j}} c_{i j} \\
& =d u_{j} c_{i j}
\end{aligned}
$$


Suppose $z$ is a solution to $\tilde{B} z=J$. Let

$$
y_{j}=\frac{1}{d} \sum_{q \in I_{j}} z_{q}
$$

Then

$$
\begin{aligned}
\sum_{j} b_{i j} y_{j} & =\sum_{j} \frac{d c_{i j}}{u_{i}} \frac{1}{d} \sum_{q \in I_{j}} z_{q} \\
& =\sum_{j} \sum_{q \in I_{j}} \frac{c_{i q} z_{q}}{u_{i}}=\sum_{q} \frac{c_{i q} z_{q}}{u_{i}} \\
& =\sum_{p \in I_{i}} \sum_{q} \frac{\tilde{b}_{p q} z_{q}}{u_{i}}=\sum_{p \in I_{i}} \frac{1}{u_{i}}=1 .
\end{aligned}
$$

Therefore $y$ is a solution to $B y=J$, and

$$
d \omega(M)=d \sum_{j} y_{j}=\sum_{q} z_{q}=\omega(\tilde{M})
$$

Proposition 6.5 Suppose $\varphi: \tilde{M} \rightarrow M$ is a d-fold regular covering. If By $=J$ has a solution, then $\tilde{B} z=J$ also has a solution.

Proof. Since $B$ is symmetric, by Lemma $6.3, \sum_{q \in I_{j}} \tilde{b}_{p q}$ is independent of the choice of $p \in I_{i}$. Therefore

$$
\sum_{q \in I_{j}} \tilde{b}_{p q}=\sum_{p \in I_{i}} \sum_{q \in I_{j}} \tilde{b}_{p q} / u_{i}
$$

For $q \in I_{j}$, let $z_{q}=d y_{j} / u_{j}$. Suppose $p \in I_{i}$. Then

$$
\begin{aligned}
\sum_{q} \tilde{b}_{p q} z_{q} & =d \sum_{q} \tilde{b}_{p q} y_{j} / u_{j}=d \sum_{j}\left(\sum_{q \in I_{j}} \tilde{b}_{p q}\right) y_{j} / u_{j} \\
& =d \sum_{j}\left(\sum_{p \in I_{i}} \sum_{q \in I_{j}} \tilde{b}_{p q} / u_{i}\right) y_{j} / u_{j} \\
& =\sum b_{i j} y_{j}=1 .
\end{aligned}
$$

Theorem 6.6 Suppose $\tilde{M} \rightarrow M$ is a d-fold covering over a nontrivial graph manifold. Then

$$
\omega(\tilde{M})=d \omega(M)
$$


Proof. By the above two propositions, the theorem is true if it is a regular covering. (In particular $\omega(M)=0$ if and only if $\omega(\tilde{M})=0$.) The general case follows by passing to a common regular covering of $M$ and $\tilde{M}$.

Corollary 6.7 If $\omega(M) \neq 0$, then the degree of a covering over $M$ is determined by the manifolds, i.e. $M$ has Property $C$.

\section{$7 \quad$ Some examples}

Example 1. The simplest graph manifolds are those whose graphs have only one vertex. Since $M$ is not Seifert fibered, there must be some edges. Therefore the matrix $B(M)$ has only one entry $b_{11} \neq 0$, and

$$
\begin{aligned}
\omega(M) & =1 / b_{11}=1 /\left(2 \sum \frac{1}{a(S) x(v)^{2}}\right) \\
& =x(v)^{2} /\left(2 \sum \frac{1}{a(S)}\right)
\end{aligned}
$$

where the sum is taken over all the components in a decomposing surface.

Example 2. The graph $\Gamma(M)$ of $M$ has two vertices. In this case there are three possibilities for the reduced graph $\Gamma^{\prime}$ of $\Gamma(M)$, according to the number its of edges.

If $\Gamma^{\prime}$ has only one edge, then $b_{12} \neq 0$, and $b_{11}=b_{22}=0$. We have

$$
\omega(M)=2 / b_{12}=2 x\left(v_{1}\right) x\left(v_{2}\right) / \sum \frac{1}{\Delta(S)}
$$

where the sum is taken over all surfaces in a decomposing surface of $M$.

Suppose $\Gamma^{\prime}$ has two edges. Without loss of generality we may assume that $b_{22}=0$. Thus the matrix is

$$
B(M)=\left(\begin{array}{cc}
b_{11} & b_{12} \\
b_{12} & 0
\end{array}\right)
$$

In this case the matrix is also nonsingular, and a solution to $B x=J$ is given by $x_{1}=1 / b_{12}$ and $x_{2}=\left(b_{12}-b_{11}\right) / b_{12}^{2}$. Hence

$$
\omega(M)=\frac{1}{b_{12}}+\frac{b_{12}-b_{11}}{b_{12}^{2}}=\frac{2 b_{12}-b_{11}}{b_{12}^{2}}
$$

which is nonzero unless $b_{11}=2 b_{12}$. 
When $\Gamma^{\prime}$ has three edges, all $b_{i j}$ are nonzero. If $\operatorname{det} B=b_{11} b_{22}-b_{12}^{2} \neq 0$, we have

$$
\omega(M)=\frac{b_{11}+b_{22}-2 b_{12}}{b_{11} b_{22}-b_{12}^{2}}
$$

If $\operatorname{det} B=0$, then $\omega(M)=0$ unless $b_{11}=b_{22}=b_{12}$, in which case $\omega(M)=1 / b_{11}$.

Example 3. The reduced graph of $\Gamma(M)$ is a chain, i.e $b_{i j}=0$ unless $i=j \pm 1$.

Let $n$ be the number of vertices. If $n$ is even, then $\operatorname{det} B(M)=b_{12}^{2}+b_{34}^{2}+\ldots+b_{n-1, n}^{2}$. The $\omega$ invariant can be computed explicitly. For example, when $n=6$,

$$
\omega(M)=2\left(\frac{1}{b_{12}}+\frac{1}{b_{34}}+\frac{1}{b_{56}}-\frac{b_{23}}{b_{12} b_{34}}-\frac{b_{45}}{b_{34} b_{56}}+\frac{b_{23} b_{45}}{b_{12} b_{34} b_{56}}\right)
$$

If $n$ is odd, $\operatorname{det} B(M)=0$, so $\omega(M)=0$ unless

$$
\operatorname{det}\left(\begin{array}{ccccc}
b_{12} & & & \cdots & 1 \\
b_{23} & b_{34} & & \cdots & 1 \\
& b_{45} & b_{56} & \ldots & 1 \\
& & b_{67} & \cdots & 1 \\
\ldots & \ldots & \ldots & \cdots & \ldots
\end{array}\right)=0
$$

Example 4. Let $f: S \rightarrow S$ be an orientation preserving homeomorphism of a compact orientable surface $S$. $f$ is called reducible if there is a nontrivial simple closed curve $C$ so that $f(C)$ is isotopic to $C$. Otherwise $f$ is irreducible. A set $\mathcal{C}=C_{1} \cup \ldots \cup C_{k}$ of mutually disjoint, mutually nonparallel, nontrivial simple closed curves on $S$ is called a canonical reducing set of $f$ if

(a) $f(\mathcal{C})$ is isotopic to $\mathcal{C}$. We can thus choose a map $g$ isotopic to $f$ with $g(\mathcal{C})=\mathcal{C}$. Then for some $n, g^{n}$ maps each component $S_{i}$ of $S-\mathcal{C}$ to itself.

(b) For each $i, g^{n} \mid S_{i}$ is either irreducible or is isotopic to a map of finite order.

(c) the number of curves in $\mathcal{C}$ is minimal subject to (a) and (b).

It is known that $\mathcal{C}$ is unique up to isotopy [12]. The mapping torus $M=M_{f}=$ $S \times I /\{(x, 0)=(f(x), 1)\}$ is a graph manifold if and only if $\mathcal{C}$ is nonempty, and $g^{n} \mid S_{i}$ is isotopic to a map of finite order for each $i$. Since $M_{f}$ is determined by the isotopy class of $f$, we may assume that $f(\mathcal{C})=\mathcal{C}$, and $f$ is periodic on $S-N(\mathcal{C})$. The image of $\mathcal{C} \times I$ in $M$ is then the canonical splitting surface of $M$. Since $M$ contains no twisted $I$-bundles, it is also the decomposing surface.

To compute $\omega(M)$, notice that $M_{f^{n}}$ is an $n$-fold covering of $M$. By the covering property of $\omega$, we need only to compute $\omega\left(M_{f^{n}}\right)$. Therefore without loss of generality we 
may assume that $f$ is an identity map on $S-N(\mathcal{C})$. Hence it is a composition of Dehn twists on the $C_{i}$ 's. Let $\tau_{i}$ be a Dehn twist on the curve $D_{i}$. Then $f=\tau_{1}^{n_{1}} \ldots \tau_{k}^{n_{k}}$, and all the $n_{i}$ 's are nonzero because $\mathcal{C}$ is minimal by (c). Now each component $S_{i}$ of $S-\mathcal{C}$ corresponds to a vertex $v_{i}$ of $\Gamma(M)$, with weight $x\left(v_{i}\right)=\chi\left(S_{i}\right)$, and each curve $C_{r}$ incident to $S_{i}$ and $S_{j}$ corresponds to an edge $e_{r}$ connecting $v_{i}$ to $v_{j}$, with weight $\Delta\left(e_{r}\right)=\left|n_{r}\right|$. The $\omega$-invariant of $M$ is now easily computed from $\Gamma(M)$. For example, if $S$ is a genus 2 closed surface and $f$ is a single Dehn twist along a nontrivial separating curve, then $\Gamma(M)$ has two vertices and one edge, $b_{12}=1$, and $\omega(M)=2$.

\section{Self coverings and cohopficity}

The $\omega$-invariant determines the covering degrees as long as it is nonzero. But the matrix $B(M)$ contains more information. We have the following

Conjecture: The degree of a covering map $\varphi: \tilde{M} \rightarrow M$ is determined by $B(\tilde{M})$ and $B(M)$.

Here is an example to show that some other invariants induced from $B(M)$ may help to determine the degrees of covering maps.

Let $v(M)$ be the number of vertices in $\Gamma(M)$. Define $b(M)=\sum b_{i j}$, where $b_{i j}$ are the entries of $B(M)$, and the sum is taken over all the entries. Suppose $\varphi: \tilde{M} \rightarrow M$ is a regular covering map, and suppose $v(\tilde{M})=v(M)$. Then each vertex of $\Gamma(M)$ is covered by exactly one vertex of $\Gamma(\tilde{M})$. In other words, $u_{i}=\left|I_{i}\right|=1$ for all $i$. Theorem 6.1 now reduces to $d \tilde{b}_{i j}=b_{i j}$, where $\tilde{b}_{i j}$ are the entries of $B(\tilde{M})$. Therefore, we have

Proposition 8.1 Suppose $\varphi: \tilde{M} \rightarrow M$ is a regular covering map over a nontrivial graph manifold. If $v(\tilde{M})=v(M)$, then the covering degree is given by $d=b(M) / b(\tilde{M})$.

Consider a (possibly non-regular) covering $\varphi: \tilde{M} \rightarrow M$. Let $\tilde{\mathcal{T}}$ and $\mathcal{T}$ be the decomposing surfaces of $\tilde{M}$ and $M$ respectively. In the proof of Proposition 5.1 we needed to use covering transformations to show that some of the weights of the graph $\Gamma(\tilde{M})$ are repeated. However, if $\tilde{\mathcal{T}}$ and $\mathcal{T}$ have the same number of tori and the same number of Klein bottles, then every torus (resp. Klein bottle) in $\mathcal{T}$ is covered by a single torus (resp. Klein bottle) in $\tilde{\mathcal{T}}$, so we do not need to use covering transformations. Hence the conclusion of 
Proposition 5.1 is still true in this setting, and is reduced to

$$
d=\frac{\Delta(\tilde{e}) \tilde{x}_{i} \tilde{x}_{j}}{\Delta(e) x_{i} x_{j}}=\frac{a(e)}{a(\tilde{e})}
$$

Summing over all edges gives $d=b(M) / b(\tilde{M})$. In particular we have

Proposition 8.2 If $M$ is a 3-manifold in $\mathcal{G}$, then it admits no covering maps of degree $>1$ over itself.

This result has also been obtained in [11]. The following lemmas will be used to prove the converse for geometric manifolds. Lemma 8.3 is essentially due to Gonzales-Acuna, Litherland and Whitten [2]. For Nil manifolds, Lemma 8.5 has also been proved in [2] with different method.

In the rest of this section (only), we will consider nonorientable manifolds as well.

Lemma 8.3 Suppose $M$ is either geometric, or is nonorientable and its orientable double covering is geometric. If $M$ is covered by (surface) $\times S^{1}$, it admits nontrivial self coverings.

Proof. In this case, the Euler number $e$ of the fibration is 0. First suppose $M$ is closed. If $M$ is orientable, by [9, $\S 4], M$ admits one of the geometric structures of $S^{2} \times R, E^{3}$ and $H^{2} \times R$. If $M$ is nonorientable, by [8] and the references there, $M$ also admits such a geometric structure. By the discussion in $[9, \S 4], M$ has the structure of a surface bundle over a 1-dimensional orbifold, so there is a two sided surface $F$ cutting $M$ into one or two (possibly twisted) $I$-bundles over surfaces. We notice that such a surface $F$ also exists in any compact Seifert manifold with boundary. For if a manifold $M$ is covered by $Q \times S^{1}$, where $Q$ is a compact surface, then the double $D M$ of $M$ is covered by $D Q \times S^{1}$, so by the above argument $D M$ has a two sided embedded surface $F^{\prime}$ transverse to all fibers. Let $F$ be a component of $F^{\prime} \cap M$. Then $F$ cuts $M$ into one or two $I$-bundles over some surfaces.

Fix the surface $F$ above. Notice that if an $I$-bundle $M_{1}$ over surface has $F$ as a boundary component, then either $M_{1}$ or its double cover is homeomorphic to $F \times I$. Therefore $M$ is covered by some $F$-bundle $\tilde{M}=F \times I / f$. Since the $I$-bundle structure arises from a Seifert fibration, the gluing map $f$ is periodic. After passing to another covering if necessary, we may assume $f$ is the identity map, i.e it sents $(x, 0)$ to $(x, 1)$, and the covering map $\varphi: \tilde{M} \rightarrow M$ sends $(x, i)$ to $x \in F, i=0,1$. Let $p: F \times I \rightarrow \tilde{M}$ be the quotient map. Now it is easy to construct a self covering of $M$ : Cut along $F$ to get a 
manifold $N$. Gluing $\partial(F \times I)$ to the two copies of $F$ on $\partial N$ by the identity map, we get a manifold $M^{\prime}=(N \cup F \times I / \cong)$ homeomorphic to $M$. The covering map $M^{\prime} \rightarrow M$ is given by mapping $N$ to $M$ by the identity map, and mapping $F \times I$ to $M$ by the composition $\varphi \circ p$.

We refer the readers to [9] for definitions and properties of Sol or Nil manifolds. The following result supplements [9, Theorem 5.3]

Lemma 8.4 If $M$ is a nonorientable Sol manifold, then it is either a union of two twisted I-bundles over a torus along their boundaries, or is a torus bundle over $S^{1}$.

Proof. By [9, Theorem 5.3], a Sol manifold is either a bundle over $S^{1}$ with fiber the torus or Klein bottle, or is the union of two twisted $I$-bundles over the torus or Klein bottle.

Notice that up to isotopy a Klein bottle $K$ has only one orientation preserving nonseparating simple closed curve, say $\alpha$. (It represents the torsion element in $H_{1}(K)$.) Thus, if $f$ is a homeomorphism of $K$, then up to isotopy we may assume that $f(\alpha)=\alpha$. If $M$ is a Klein bottle bundle over $S^{1}$, then $M=K \times I / f$, where $f: K \times 0 \rightarrow K \times 1$ is a homeomorphism. We may assume that $f(\alpha \times 0)=\alpha \times 1$. Thus the $S^{1}$-fibration of $K \times I$ by circles parallel to $\alpha$ gives rise to a Seifert fibration of $M$. This is impossible because a Sol manifold does not admit Seifert fibrations.

Similarly, if $M=M_{1} \cup_{f} M_{2}$, with $M_{i}$ twisted $I$-bundles over a Klein bottle such that the $\partial M_{i}$ are Klein bottles, then the orientation preserving nonseparating simple closed curves $\alpha_{i}$ extend to Seifert fibrations on $M_{i}$, and $f$ maps $\alpha_{1}$ to $\alpha_{2}$. Thus $M$ is Seifert fibered, again a contradiction.

In the remaining cases, $M$ is either orientable, or is a torus bundle over a 1-orbifold. This completes the proof.

Lemma 8.5 If a 3-manifold $M$ is covered by some torus bundle over $S^{1}$, then it admits nontrivial self coverings.

Proof. If the gluing map of the torus bundle is periodic, $M$ is also covered by (surface) $\times S^{1}$, so the result follows from Lemma 8.3. Otherwise $M$ admits either a Nil or Sol structure. Any Nil manifold is orientable because the Euler number of its Seifert bundle is nonzero. By [9, Theorem 5.3] and Lemma 8.4, we have only three possibilities: (1) $M$ is a torus 
bundle over $S^{1}$; (2) $M=M_{1} \cup_{f} M_{2}$, where the $M_{i}$ are orientable, and are twisted $I$-bundles over a Klein bottle; (3) $M=M_{1} \cup_{f} M_{2}$, where $M_{i}$ are twisted $I$-bundles over a torus.

In Case 1, we have $M=T \times I / f$, where $f: T \times 0 \rightarrow T \times 1$ is a homeomorphism. Let $\varphi: T \times I \rightarrow T \times I$ be a self covering such that $\varphi_{*}(x)=x^{p}$ for all $x \in \pi_{1}(T \times I)$, where $p>1$ is a positive integer. Then

$$
f_{*} \varphi_{*}(x)=f_{*}\left(x^{p}\right)=\left(f_{*}(x)\right)^{p} \in \varphi_{*}\left(\pi_{1}(T \times 1)\right)
$$

i.e $f_{*} \varphi_{*} \pi_{1}(T \times 0)$ is a subgroup of $\varphi_{*} \pi_{1}(T \times I)$. Since $f_{*}: \pi_{1}(T \times 0) \rightarrow \pi_{1}(T \times 1)$ is an isomorphism, by checking the indices of subgroups, one can see that $f_{*}: \varphi_{*} \pi_{1}(T \times 0) \rightarrow$ $\varphi_{*} \pi_{1}(T \times 1)$ is actually an isomorphism. Thus $f$ can be lifted to a homeomorphism $\tilde{f}: T \times 0 \rightarrow T \times 1$ so that $\varphi \circ \tilde{f}=f \circ \varphi$ on $T \times 0$. Hence $\varphi$ induces a covering map $\bar{\varphi}: T \times I / \tilde{f} \rightarrow T \times I / f$. Also, for all $x \in \pi_{1}(T \times 0)$,

$$
\varphi_{*} \tilde{f}_{*}(x)=f_{*} \varphi_{*}(x)=f_{*}\left(x^{p}\right)=\left(f_{*}(x)\right)^{p}=\varphi_{*}\left(f_{*}(x)\right)
$$

As $\varphi_{*}$ is injective, $\tilde{f}_{*}=f_{*}$, so $\tilde{f}$ is isotopic to $f$. It follows that $T \times I / \tilde{f}$ is homeomorphic to $M$.

In Case 2, let $\pi_{1}\left(M_{i}\right)=<a_{i}, b_{i} \mid a_{i} b_{i} a_{i}^{-1}=b_{i}^{-1}>$, where $a_{i}, b_{i}$ are represented by simple closed curves on the central Klein bottle of $M_{i}$. For odd integers $p>1$, one can construct $p^{2}$ fold coverings $\varphi_{i}: M_{i} \rightarrow M_{i}$ so that $\varphi_{*}\left(a_{i}\right)=a_{i}^{p}$ and $\varphi_{*}\left(b_{i}\right)=b_{i}^{p}$. Let $\alpha_{i}, \beta_{i}$ be generators of $\pi_{1}\left(\partial M_{i}\right)$ such that $j_{*}\left(\alpha_{i}\right)=a_{i}^{2}$, and $j_{*}\left(\beta_{i}\right)=b_{i}$, where $j: \partial M \rightarrow M$ is the inclusion map. Denote by $\psi_{i}$ the restriction of $\varphi_{i}$ on $\partial M_{i}$. Then $\psi_{i *}\left(\alpha_{i}\right)=\alpha_{i}^{p}$, and $\psi_{i *}\left(\beta_{i}\right)=\beta_{i}^{p}$. Now we can apply the argument in Case 1 to show that $f$ lifts to a map $\tilde{f}: \partial M_{1} \rightarrow \partial M_{2}$ such that $\psi_{2} \tilde{f}=f \psi_{1}$, and $\tilde{f}$ is isotopic to $f$; so the $\varphi_{i}$ induce a covering $\operatorname{map} \bar{\varphi}: M_{1} \cup_{\tilde{f}} M_{2} \rightarrow M$, and $M_{1} \cup_{\tilde{f}} M_{2}$ is homeomorphic to $M$.

The proof of Case 3 is similar to that of Case 2. In this case $M$ admits self coverings of degree $p^{2}$ for all $p \geq 1$.

Theorem 8.6 Suppose $M$ is either geometric, or is nonorientable and is double covered by some geometric manifold. Then $M$ admits a nontrivial self covering if and only if it is covered by (surface) $\times S^{1}$ or a torus bundle over $S^{1}$.

Proof. The orientable case follows directly from Proposition 8.2, Lemma 8.4 and Lemma 8.5. So suppose $M$ is nonorientable. If $M$ admits a nontrivial self covering, its orientable 
double cover also does, so the necessity follows from (1). The sufficiency follows from Lemmas 8.3 and 8.5 .

The above results can be used to answer the cohopficity problem of closed geometric manifold groups. A group $G$ is called cohopfian if it is not a proper subgroup of itself. In [1] Gonzales-Acuna and Whitten studied the cohopficity of 3-manifold groups. They solved this problem for a Haken manifold $M$ with boundary a nonempty union of tori, and asked what closed irreducible 3-manifolds have infinite cohopfian groups. The reason for restricting to irreducible manifolds is as follows. If $M=S^{1} \times S^{2}$, then $\pi_{1}(M)=\mathbf{Z}$ is clearly not cohopfian. If $M=M_{1} \# M_{2}$ and $\pi_{1}\left(M_{i}\right) \neq 1$, then $\pi_{1}(M)=\pi_{1}\left(M_{1}\right) * \pi_{1}\left(M_{2}\right)$. Pick nontrivial elements $\alpha \in \pi_{1}\left(M_{1}\right)$ and $\beta \in \pi_{1}\left(M_{2}\right)$. Then $\pi_{1}(M)$ has a proper subgroup $\left(\pi_{1}\left(M_{1}\right)\right)^{\beta} *\left(\pi_{1}\left(M_{2}\right)\right)^{\alpha}$ isomorphic to $\pi_{1}(M)$ itself, where $G^{x}$ represents the subgroup $\left\{x g x^{-1} \mid g \in G\right\}$. Therefore $\pi_{1}(M)$ is not cohopfian. Note also that if $\pi_{1}\left(M_{2}\right)=1$, then $\pi_{1}(M)=\pi_{1}\left(M_{1}\right)$, so $\pi_{1}(M)$ is cohopfian if and only if $\pi_{1}\left(M_{1}\right)$ is.

In [2] Gonzales-Acuna, Litherland and Whitten answered the above question for Seifert fibered manifolds. The group of a Seifert bundle is cohopfian if and only if it is not covered by (surface) $\times S^{1}$ or torus bundle over $S^{1}$. Using Theorem 8.6, we can show that this is also true for all irreducible geometric manifolds.

Theorem 8.7 If $M$ is a closed geometric 3-manifold, then $\pi_{1}(M)$ is cohopfian if and only if $M$ is irreducible and is not covered by (surface) $\times S^{1}$ or torus bundle over $S^{1}$.

Proof. As remarked before, we may assume $M$ is irreducible. By [2], the result is true for Seifert fibered spaces. So we may assume that $M$ is either hyperbolic or is a Haken manifold. If $M$ is covered by (surface) $\times S^{1}$ or torus bundle over $S^{1}$, then by Theorem 8.6, $M$ admits nontrivial self coverings, so it is not cohopfian.

Suppose $G$ is a proper subgroup of $\pi_{1} M$ which is isomorphic to $\pi_{1} M$. Let $\varphi: \tilde{M} \rightarrow M$ be the covering map corresponding to $G$. Then $M, \tilde{M}$ have the same fundamental group. Since $H_{3}(G)=H_{3}(M), \tilde{M}$ is closed. It is well known that closed hyperbolic or Haken manifolds are determined by their fundamental groups. Therefore $\varphi$ is a nontrivial self covering of $M$. It follows from Theorem 8.6 that $M$ is covered by (surface) $\times S^{1}$ or torus bundle over $S^{1}$.

Proposition 8.8 Let $M$ be an orientable irreducible 3-manifold that double covers a closed nonorientable manifold $N$. If $\pi_{1} M$ is cohopfian, so is $\pi_{1} N$. 
Proof. Suppose $\pi_{1} N$ is not cohopfian. Let $G$ be a proper subgroup of $\pi_{1} N$, and let $\varphi: G \rightarrow \pi_{1} N$ be an isomorphism. We may assume $\pi_{1} N$ is infinite, otherwise it is cohopfian. Since $M$ is irreducible, $N$ is aspherical, so we have $H_{3}\left(G ; \mathbf{Z}_{2}\right)=H_{3}\left(N ; \mathbf{Z}_{2}\right) \neq 0$, (see [4] for homology of groups.) Thus the covering space of $N$ corresponding to $G$ is closed, and hence $G$ is of finite index in $\pi_{1} N$.

Consider $\pi_{1} M$ as a subgroup of index 2 in $\pi_{1} N$. Let $G_{+}=G \cap \pi_{1} M$. Since $G_{+}$is a subgroup of finite index in $\pi_{1} M$, it is the group of some closed orientable aspherical 3-manifold, so $H_{3}\left(G_{+} ; \mathbf{Z}\right)=\mathbf{Z}$. Since $H_{3}(G ; \mathbf{Z})=H_{3}(N ; \mathbf{Z})=0$, we have $G_{+} \neq G$, i.e $G_{+}$ is a proper subgroup of $G$. As $\pi_{1} M$ has index 2 in $\pi_{1} N, G_{+}=G \cap \pi_{1} M$ has index 2 in $G$.

Notice that $\varphi\left(G_{+}\right)$has index 2 in $\pi_{1} N=\varphi(G)$. If it is not contained in $\pi_{1} M$, then it contains some elements represented by orientation reversing curves of $N$, so the covering space $\tilde{M}$ corresponding to $\varphi\left(G_{+}\right)$is nonorientable. This is impossible because $H_{3}(\tilde{M} ; \mathbf{Z})=$ $H_{3}\left(G_{+} ; \mathbf{Z}\right)=\mathbf{Z}$, as shown above. Therefore $\varphi\left(G_{+}\right) \subset \pi_{1} M$. Since they have the same index in $\pi_{1} N, \varphi\left(G_{+}\right)=\pi_{1} M$. Thus $\varphi$ induces an isomorphism $G_{+} \cong \pi_{1} M$. Use $\left[\pi_{1} N: G_{+}\right]$ to denote the index of $G_{+}$in $\pi_{1} N$. Then

$$
\left[\pi_{1} N: G_{+}\right]=\left[\pi_{1} N: \pi_{1} M\right]\left[\pi_{1} M: G_{+}\right]=\left[\pi_{1} N: G\right]\left[G: G_{+}\right]
$$

As $\left[\pi_{1} N: \pi_{1} M\right]=\left[G: G_{+}\right]=2$, we have $\left[\pi_{1} M: G_{+}\right]=\left[\pi_{1} N: G\right] \neq 1$, so $G_{+}$is a proper subgroup of $\pi_{1} M$, contradicting the assumption that $\pi_{1} M$ is cohopfian.

Theorem 8.9 If a closed nonorientable 3-manifold $N$ is double covered by an irreducible geometric 3-manifold $M$, then $\pi_{1} N$ is cohopfian if and only if $M$ is not covered by (surface) $\times S^{1}$ or torus bundle over $S^{1}$.

Proof. if $N$ is not covered by (surface) $\times I$ or torus bundle over $S^{1}$, then $M$ is not either, so by Theorem 8.7 and Proposition $8.8, \pi_{1}(N)$ is not cohopfian. If $N$ is covered by (surface) $\times I$ or torus bundle over $S^{1}$, it admits nontrivial self covering by Theorem 8.6, so $\pi_{1}(N)$ is not cohopfian.

We do not know whether $\pi_{1} N$ is cohopfian if $N$ is double covered by a reducible manifold. 


\section{Symmetries of the B-matrix}

In this section we study the symmetry of the matrix $B(M)$. This will provide some useful information in determining the covering degrees. Notice that when $\Gamma(M)$ has only one vertex, the $\omega$ invariant of $M$ is a positive number, so $M$ has property $\mathrm{C}$. This method does not work for some graph manifolds with more than one vertex, but we will use symmetry to determine the degrees of regular covering maps over graph manifolds with at most 3 vertices.

To clarify the idea, we define a weighted graph $L(M)$ as follows. $L(M)$ has the same vertices as $\Gamma(M)$, and it has one edge $e_{i j}$ for each pair of vertices $v_{i}, v_{j}$ ( $i$ may equal to $j$ ). Assign $b_{i j}$ as the weight of $e_{i j}$. An isometry $g$ of $L(M)$ is an automorphism of the graph which preserves the weights, i.e the weight of $e_{i j}$ equals that of $g\left(e_{i j}\right)$ for all $i, j$. Denote the isometry group of $L(M)$ by $G(M)$. It induces an action on the set $V(M)$ of vertices of $L(M)$. We denote by $[v]_{G}$ the orbit of $v$ under this action, by $n(v)$ the number of elements in the orbit $[v]_{G}$, and by $O_{G}(M)=|V(M) / G(M)|$ the number of orbits in $V(M)$.

Suppose $\varphi: \tilde{M} \rightarrow M$ is a regular covering map. A covering transformation $f: \tilde{M} \rightarrow \tilde{M}$

induces a map $f_{\#}: L(\tilde{M}) \rightarrow L(\tilde{M})$ which is clearly an isometry. Let $F(\tilde{M})$ be the set of isometries induced by covering transformations. Then $F(\tilde{M})$ is a subgroup of $G(\tilde{M})$. Since $\varphi$ is a regular covering, it induces a one to one correspondence $V(\tilde{M}) / F(\tilde{M}) \rightarrow V(M)$. Since $F(\tilde{M})$ is a subgroup of $G(\tilde{M})$, for each vertex $v \in V(M), \varphi^{-1}(v)$ is contained in a $G$-orbit of $V(\tilde{M})$. In other words, if $\tilde{v}$ covers $v$, then $\varphi^{-1}(v) \subset[\tilde{v}]_{G}$.

Define

$$
c(M)=\sum_{i, j} \frac{b_{i j}}{n\left(v_{i}\right) n\left(v_{j}\right)} .
$$

Clearly, this is an invariant of $M$.

Theorem 9.1 Suppose $\varphi: \tilde{M} \rightarrow M$ is a regular covering over a nontrivial graph manifold. If $O_{G}(\tilde{M})=v(M)$, then $d c(\tilde{M})=b(M)$.

Proof. Recall that $b(M)=\sum b_{i j}$. By Theorem 6.1 we have

$$
d \sum_{p \in I_{i}} \sum_{q \in I_{j}} \tilde{b}_{p q}=b_{i j} u_{i} u_{j}
$$

where $u_{i}$ is the number of vertices in $L(\tilde{M})$ that cover $v_{i}$. Since $O_{G}(\tilde{M})=v(M)$, each $G$-orbit covers only one vertex of $L(M)$. Therefore, $u_{i}=n\left(\tilde{v}_{p}\right)$ if $p \in I_{i}$. Now the above 
formula can be written as

$$
d \sum_{p \in I_{i}} \sum_{q \in I_{j}} \frac{\tilde{b}_{p q}}{n\left(\tilde{v}_{p}\right) n\left(\tilde{v}_{q}\right)}=b_{i j} .
$$

Summing over all $i, j$, we get the required formula $d c(\tilde{M})=b(M)$.

In general, we have $1 \leq O_{G}(\tilde{M}) \leq v(M)$. Since $b(M) \neq 0$, the above theorem determines the degree $d$ in one extreme case. The following result shows that the $\omega$ invariant determines $d$ in the other extreme case.

Theorem 9.2 If $O_{G}(M)=1$, then $\omega(M)=v(M)^{2} / b(M)$.

Proof. By definition, for any $i, j$, there is an isometry $g \in G(M)$ sending $v_{i}$ to $v_{j}$. Suppose $g$ maps $v_{k}$ to $v_{g(k)}$. Since $g$ is an isometry, we have $b_{i k}=b_{j g(k)}$. When $v_{k}$ ranges over all vertices of $L(M), v_{g(k)}$ also ranges over all vertices. Therefore,

$$
\sum_{k} b_{i k}=\sum_{k} b_{j g(k)}=\sum_{k} b_{j k}
$$

that is, $c=\sum_{k} b_{i k}$ is a constant. Since $B(M)$ is a nonzero matrix, $c$ is nonzero. It follows that $x^{T}=(1 / c, \ldots, 1 / c)^{T}$ is a solution to $B(M) x=J$. Therefore, $\omega(\tilde{M})=\sum x_{i}=$ $v(M) / c=v(M)^{2} / \sum b_{i j}=v(M)^{2} / b(M)$.

Corollary 9.3 Suppose $\varphi: \tilde{M} \rightarrow M$ is a regular covering over a nontrivial graph manifold. If $O_{G}(\tilde{M})=1$, then $\omega(\tilde{M}) \neq 0$; hence $d=\omega(\tilde{M}) / \omega(M)$.

Theorem 9.4 Suppose $\varphi: \tilde{M} \rightarrow M$ is a regular covering over a nontrivial graph manifold. If $v(M) \leq 3$, then the degree of $\varphi$ is determined by $\tilde{M}$ and $M$.

Proof. By the above discussion, if $O_{G}(\tilde{M})=1$ or $v(M)$, then the result is true; so we may assume $v(M)=3$ and $O_{G}(\tilde{M})=2$.

Let $\tilde{V}_{i}$ be the set of vertices in $L(\tilde{M})$ that cover $v_{i}$. Since $O_{G}(\tilde{M})=2$, without loss of generality we may assume that $\tilde{V}_{1}$ is a $G$-orbit, and $\tilde{V}_{2} \cup \tilde{V}_{3}$ is the other one. Let $\tilde{v}_{p}, \tilde{v}_{q} \in \tilde{V}_{2} \cup \tilde{V}_{3}$ be any two vertices. By assumption there is an isometry $g$ sending $\tilde{v}_{p}$ to $\tilde{v}_{q}$. Since $\tilde{V}_{1}$ is an orbit, $g$ induces a permutation on $\tilde{V}_{1}$. Therefore,

$$
\sum_{r \in I_{1}} \tilde{b}_{p r}=\sum_{r \in I_{1}} \tilde{b}_{q r}=c
$$


is a constant. Thus we have

$$
b_{12} u_{1} u_{2}=d \sum_{p \in I_{2}} \sum_{r \in I_{1}} \tilde{b}_{p r}=d \sum_{p \in I_{2}} c=d u_{2} c
$$

so $b_{12}=d c / u_{1}$. Similarly, we have $b_{13}=d c / u_{1}=b_{12}$.

To compute the degree, we consider the subgraph $L^{\prime}(\tilde{M})$ consisting of all edges with one endpoint in $\tilde{V}_{1}$ and the other in $\tilde{V}_{2} \cup \tilde{V}_{3}$, and in $L(M)$ consider $L^{\prime}(M)=e_{12} \cup e_{13}$. Since $G(\tilde{M})$ is determined by $\tilde{M}$, we see that $L^{\prime}(\tilde{M})$ is determined by $\tilde{M}$. If $b_{23} \neq b_{12}=b_{13}$, then $L^{\prime}(M)$ is also well defined. In other words, one can "see" the subgraph directly from $L(M)$ without appealing to $\varphi$. If $b_{23}=b_{12}=b_{13}$, we can pick up any two edges, and the weighted graph is always the same. Therefore, as a weighted graph $L^{\prime}(M)$ is also well defined. That is, it is independent of $\varphi$, as long as $O_{G}(\tilde{M})=2$.

Define $\omega\left(L^{\prime}(M)\right)$ in the same manner as in the definition of $\omega(M)$. Actually, one can see that if we use $M^{\prime}$ to denote the manifold obtained from $M$ by cutting along those surfaces in the decomposing surface of $M$ that does not correspond to the edges $e_{12}$ or $e_{13}$, then $\omega\left(L^{\prime}(M)\right)=\omega\left(M^{\prime}\right)$. Thus the covering property is still true, and we have

$$
d \omega\left(L^{\prime}(\tilde{M})\right)=\omega\left(L^{\prime}(M)\right)
$$

Since $\omega\left(L^{\prime}(M)\right)=2 / b_{12} \neq 0$, the degree $d$ is determined by $M$ and $\tilde{M}$.

Acknowledgement. We would like to thank Professor C.T.C. Wall and the referee for many useful comments.

\section{References}

[1] F. Gonzales-Acuna and W. Whitten: Imbeddings of 3-manifold groups, Mem. Amer. Math. Soc. (to appear).

[2] F. Gonzales-Acuna, R. Litherland and W. Whitten: Cohopficity of Seifert bundle groups, preprint.

[3] M. Gromov: Volume and bounded cohomology, Publ. Math. IHES, 56 (1983) 5-99.

[4] J. Hempel: 3-manifolds, Ann. of Math. Study 86, Princeton University Press 1976.

[5] W. Jaco: Lectures on three-manifold topology, Amer. Math. Soc. regional conference series in mathematics 43 (1980).

[6] W. Jaco and P. Shalen: Seifert fibered spaces in 3-manifolds, Mem. Amer. Math. Soc. 220 (1979).

[7] R. Kirby: Problems in low dimensional manifold theory, Algebraic and Geometric topology, (ed. by R.J. Milgram), Proc. Symposia in Pure Math. 32 (1978) 273-312, Amer. Math. Soc. 
[8] W. Meeks and P. Scott: Finite group actions on 3-manifolds, Invent. Math. 86 (1986) 287-346.

[9] P. Scott: The geometries of 3-manifolds, Bull. London Math. Soc. 15 (1983) 401-487.

[10] W. Thurston: Three dimensional manifolds, Kleinian groups and hyperbolic geometry, Bull. Amer. Math. Soc. 6 (1982) 357-382.

[11] S. Wang: The $\pi_{1}$-injectivity of selfmaps of nonzero degree on 3-manifolds, Math. Ann. (to appear).

[12] Y-Q. Wu: Canonical reducing curves of surface homeomorphisms, Acta Math. Sinica, (N.S.) 3 (1987) 305-313.

Department of Mathematics, Peking University, Beijing 100871, P.R.CHINA

Department of Mathematics, University of California, Santa Barbara, CA 93106 